\title{
Contact process on generalized Fibonacci chains: infinite-modulation criticality and double-log periodic oscillations
}

\author{
Hatem Barghathi, ${ }^{1}$ David Nozadze, ${ }^{1,2}$ and Thomas Vojta $^{1}$ \\ ${ }^{1}$ Department of Physics, Missouri University of Science and Technology, Rolla, MO 65409, USA \\ ${ }^{2}$ Department of Physics, The Ohio State University, Columbus, OH 43210, USA
}

(Dated: August 21, 2018)

\begin{abstract}
We study the nonequilibrium phase transition of the contact process with aperiodic transition rates using a real-space renormalization group as well as Monte-Carlo simulations. The transition rates are modulated according to the generalized Fibonacci sequences defined by the inflation rules $\mathrm{A} \rightarrow$ $\mathrm{AB}^{k}$ and $\mathrm{B} \rightarrow \mathrm{A}$. For $k=1$ and 2 , the aperiodic fluctuations are irrelevant, and the nonequilibrium transition is in the clean directed percolation universality class. For $k \geq 3$, the aperiodic fluctuations are relevant. We develop a complete theory of the resulting unconventional "infinite-modulation" critical point which is characterized by activated dynamical scaling. Moreover, observables such as the survival probability and the size of the active cloud display pronounced double-log periodic oscillations in time which reflect the discrete scale invariance of the aperiodic chains. We illustrate our theory by extensive numerical results, and we discuss relations to phase transitions in other quasiperiodic systems.
\end{abstract}

\section{INTRODUCTION}

Many-particle systems far from equilibrium can display abrupt transitions between different nonequilibrium steady states that share many characteristics with equilibrium phase transitions. Examples of such nonequilibrium phase transitions occur in turbulence, catalytic reactions, interface growth, and in the dynamics of epidemics and other biological populations [1 8].

Absorbing-state transitions constitute a particularly well-studied subclass of nonequilibrium phase transitions. They separate active, fluctuating steady states from absorbing states which are completely inactive and do not display any fluctuations. Generically, absorbingstate transitions are in the directed percolation (DP) universality class [9], provided they feature a scalar order parameter and short-range interactions but no extra symmetries or conservation laws [10, 11]. The contact process [12] is a prototypical model in the DP universality class. Experimental examples of absorbing state transitions were found in turbulent liquid crystals [13], periodically driven suspensions [14, 15], and in systems of superconducting vortices [16.

Many realistic experimental systems contain various types of spatial inhomogeneities. For this reason, the effects of such inhomogeneities on absorbing state transitions have attracted considerable attention. Random disorder was shown to destabilize the clean DP critical point [17] because its correlation length critical exponent $\nu_{\perp}$ violates the Harris criterion [18] $d \nu_{\perp}>2$ in space dimensions $d=1,2$ and 3. Early numerical simulations of the disordered contact process [19 22] showed unusually slow dynamics but the ultimate fate of the transition was only resolved by means of a strong-disorder renormalization group analysis [23] of the one-dimensional disordered contact process. It yielded an exotic infinite-randomness critical point accompanied by power-law Griffiths singularities 24]. The renormalization group predictions were confirmed by Monte-Carlo simulations [25], and analogous behavior was also found in two and three dimensions [26, 27] as well as in diluted systems at the lattice percolation threshold [28].

Spatial inhomogeneities can arise not just from random disorder but also from deterministic but aperiodic (quasiperiodic) modulations of the transition rates defining the nonequilibrium process. The stability of a clean critical point against such aperiodic fluctuations can be tested by means of a generalization of the Harris criterion, the Harris-Luck criterion [29], which relates the clean correlation length exponent $\nu_{\perp}$ and the wandering exponent $\omega$ of the aperiodic structure.

In this paper, we use a real-space renormalization group as well as Monte-Carlo simulations to study the one-dimensional contact process with aperiodic transition rates modulated according to the generalized $\mathrm{Fi}$ bonacci sequences defined by the inflation rules $\mathrm{A} \rightarrow$ $\mathrm{AB}^{k}$ and $\mathrm{B} \rightarrow \mathrm{A}$. For $k=1$ and 2 , the aperiodic fluctuations are irrelevant according to the Harris-Luck criterion. Correspondingly, we find the nonequilibrium transition to be in the clean directed percolation universality class. For $k \geq 3$, the aperiodic fluctuations are relevant. We develop a complete theory of the resulting "infinitemodulation" critical point. It is characterized by a diverging strength of the inhomogeneities and features activated dynamical scaling similar to the disordered contact process. Moreover, observables display double-log periodic oscillations in time which reflect the discrete scale invariance of the aperiodic chains. We also confirm and illustrate the renormalization group predictions by extensive numerical simulations.

The paper is organized as follows. In Sec. [II we introduce the contact process and the generalized Fibonacci chains. We also discuss the Harris-Luck criterion. The renormalization group theory is developed in Sec. III. Section [V] is devoted to the Monte-Carlo simulations. We conclude in Sec. V. 


\section{CONTACT PROCESS ON APERIODIC CHAINS}

\section{A. Generalized Fibonacci chains}

We consider a family of aperiodic two-letter sequences generated by the inflation rules

$$
\begin{aligned}
& \mathrm{A} \rightarrow \mathrm{AB}^{k} \\
& \mathrm{~B} \rightarrow \mathrm{A}
\end{aligned}
$$

where $k$ is a positive integer and $\mathrm{B}^{k}$ stands for a sequence of $k$ letters $\mathrm{B}$. The case $k=1$ corresponds to the famous Fibonacci sequence. For $k=2$, the fourthgeneration sequence (starting from a single letter A) reads $A B B A A A B B A B B$. In general, the sequences created by eq. (11) contain groups of $k$ letters B separated by either single letters A or groups of $k+1$ letters A. Many properties of these sequences can be obtained from the substitution matrix

$$
\mathbf{M}_{k}=\left(\begin{array}{ll}
1 & 1 \\
k & 0
\end{array}\right)
$$

which describes how the numbers $N_{A}$ and $N_{B}$ of letters $A$ and $B$ evolve under the inflation (see, e.g., Ref. 30] and references therein). Its eigenvalues read

$$
\zeta_{ \pm}=\frac{1}{2}(1 \pm \sqrt{1+4 k})
$$

The larger eigenvalue $\zeta_{+}$controls how the total length $N(i)=N_{A}(i)+N_{B}(i)$ increases with the inflation step $i$. In the limit of large $i$, one obtains $N_{i} \sim \zeta_{+}^{i}$. The smaller eigenvalue $\zeta_{-}$governs the fluctuations of the numbers $N_{A}$ and $N_{B}$. Specifically, $\Delta N_{A}(i)=\left|N_{A}(i)-x_{A} N(i)\right| \sim\left|\zeta_{-}\right|^{i}$ for large $i$. Here $x_{A}=\lim _{i \rightarrow \infty} N_{A}(i) / N(i)$ is the fraction of letters $A$ in the infinite chain. The same relation also holds for $N_{B}$. The wandering exponent $\omega$ relates the fluctuations to the length of the chain, $\Delta N_{A}(i) \sim N(i)^{\omega}$. This yields the equation

$$
\omega=\ln \left|\zeta_{-}\right| / \ln \zeta_{+}
$$

For the generalized Fibonacci chains defined in (11), the specific values are $\omega_{1}=-1, \omega_{2}=0$ and $\omega_{3} \approx 0.3171$ for $k=1,2$ and 3 . Upon further increasing $k, \omega$ increases monotonically and reaches 1 for $k \rightarrow \infty$.

\section{B. Contact process}

The (clean) contact process [12] is one of the simplest systems undergoing an absorbing state transition. It can be understood as model for the spreading of an epidemic. Each lattice site can be in one of two states, active (infected) or inactive (healthy). Over time, active sites can infect their neighbors or they can heal spontaneously. More precisely, the time evolution is a continuous-time Markov process during which infected sites heal at a rate

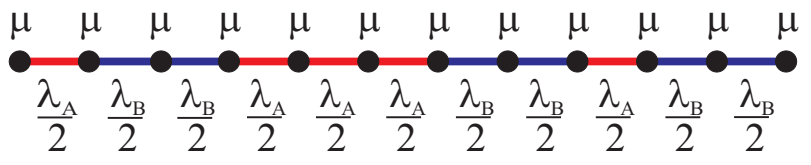

FIG. 1. (Color online) Sequence of transition rates for the contact process on a generalized Fibonacci chain, showing the 4 th generation of the $k=2$ chain.

$\mu$ while healthy sites become infected by their neighbors at a rate $\lambda n /(2 d)$. Here, $n$ is the number of sick nearest neighbors of the given site.

The long-time behavior of the system is controlled by the ratio of the infection rate $\lambda$ and the healing rate $\mu$. For $\lambda \ll \mu$, healing dominates over infection, and the epidemic eventually dies out completely. Thus, the model ends up in the absorbing state without any infected sites. This is the inactive phase. In contrast, the density of infected sites remains nonzero in the long-time limit if the infection rate $\lambda$ is sufficiently large, i.e., the model is in the active phase. The nonequilibrium transition separating these two phases belongs to the DP universality class.

Spatial inhomogeneity can be introduced into the contact process by making the infection and/or healing rates dependent on the lattice site. We are interested in aperiodic (quasiperiodic) inhomogeneities. Specifically, we consider a chain of sites that have equal healing rates $\mu$ but two different the infection rates $\lambda_{A}$ and $\lambda_{B}$ [31]. They are arranged on the bonds of the chain according to the generalized Fibonacci sequences discussed in the last section. An example $(k=2)$ is shown in Fig. 1. For $\lambda_{A}=\lambda_{B}$, the system coincides with the usual (clean) one-dimensional contact process.

\section{Harris-Luck criterion}

Luck 29] derived a criterion for the stability of a clean critical point against weak aperiodic inhomogeneities. The basic idea is analogous to that of the Harris criterion for random disorder: The clean critical point is stable of the fluctuations $\Delta r$ of the local distance from criticality between different correlation volumes are smaller than the global distance $r$ to criticality. For aperiodic inhomogeneities characterized by a wandering exponent $\omega$, the fluctuations behave as $\Delta r \sim N^{\omega-1} \sim \xi^{d(\omega-1)}$ while the global distance from criticality scales as $r \sim \xi^{-1 / \nu_{\perp}}$. The condition $\Delta r<r$ for $\xi \rightarrow \infty$ leads to the exponent inequality

$$
\omega<1-\frac{1}{d \nu_{\perp}}
$$

This is the Harris-Luck criterion. In the case of random disorder, $\omega=1 / 2$, it reduces to the usual Harris criterion [18]. If the inequality (5) is fulfilled, weak inhomogeneities are irrelevant, otherwise they are relevant and change the character of the phase transition. 
The correlation length exponent of the onedimensional clean contact process takes the value $\nu_{\perp} \approx 1.097$. The Harris-Luck criterion thus simplifies to $\omega<1-1 / \nu_{\perp} \approx 0.0884$. This implies that aperiodic fluctuations of the transition rates are irrelevant for $k=1$ and 2 while they are relevant for $k \geq 3$.

\section{REAL-SPACE RENORMALIZATION GROUP}

\section{A. Overview}

This section is devoted to a real-space renormalization group for the contact process on generalized Fibonacci chains. Our method is inspired by a similar calculation for the transverse-field Ising chain 32]. There are, however, some important differences.

Let us start by assuming that the transition rates fulfill the condition $\lambda_{A} \ll \mu \ll \lambda_{B}$. We can then perform a renormalization group step which consists of two parts:

(i) Combine the $(k+1)$ consecutive sites connected by the large $\lambda_{B}$ infection rate into a single new site with a renormalized healing rate $\tilde{\mu} \ll \mu$. Structurally, this reverses one inflation step, as the result is a system with uniform infection rates $\lambda_{A}$ but two different healing rates, $\mu$ and $\tilde{\mu}$, modulated according to a Fibonacci chain of one generation earlier.

(ii) Integrate out the sites with the original healing rate $\mu$ which is now the largest transition rate in the system. This generates renormalized infection rates (bonds) $\tilde{\lambda}$ between the remaining sites and reverses another inflation step. The system now has uniform healing rate $\tilde{\mu}$ and two different infection rates $\lambda_{A}$ and $\tilde{\lambda}$ modulated according to a Fibonacci chain of two generations earlier than the original chain.

After renaming $\lambda_{A} \rightarrow \lambda_{B}, \tilde{\lambda} \rightarrow \lambda_{A}$ and $\tilde{\mu} \rightarrow \mu$ we arrive at a system equivalent to the original one, but with renormalized transition rates. As long as the renormalized rates still fulfill the condition $\lambda_{A} \ll \mu \ll \lambda_{B}$, this renormalization group step can be iterated.

In the opposite limit, $\lambda_{A} \gg \mu \gg \lambda_{B}$, an analogous renormalization group step does not preserve the structure of the system and can thus not be iterated. However, we will study the fate of systems in this regime numerically at the end of Sec. IV] If the healing rate $\mu$ is much larger (or smaller) than both infection rates, the system can never reach criticality, instead it is deep in the inactive (or active) phase.

\section{B. Recursion relations}

We now analyze the renormalization group step outlined above in a quantitative manner. The infection rate $\lambda_{B}$ is the largest transition rate in the system. Thus, sites coupled by $\lambda_{B}$-bonds will quickly reinfect each other when one of them heals. Consequently, all $k+1$ sites coupled by the $k$ consecutive $\lambda_{B}$ bonds can be merged into a single new site of "moment" (number of sites)

$$
\tilde{m}=(k+1) m
$$

where $m$ is the moment of the original sites (in the bare system, $m=1)$. The renormalized healing rate $\tilde{\mu}$ of these new sites can be found either by directly enumerating all possible healing paths of the cluster or by analyzing the eigenvalues of the generator of the Markov process in the Hamiltonian formalism (see, e.g., Ref. [23]). Both methods give the same result,

$$
\tilde{\mu}=\alpha_{k} \frac{\mu^{k+1}}{\left(\lambda_{B} / 2\right)^{k}}
$$

with $\alpha_{1}=2, \alpha_{2}=4$, and $\alpha_{3}=8$. If $\mu \ll \lambda_{B}$, the renormalized healing rate is strongly reduced, $\tilde{\mu} \ll \mu$.

After the first part of the renormalization group step, the system has uniform infection rates $\lambda_{A}$ but two types of sites, original sites having healing rate $\mu$ and new sites having healing rate $\tilde{\mu}$. If the rates fulfill the condition $\tilde{\mu} \ll \lambda_{A} \ll \mu$, we can perform the second part of the renormalization group step and integrate out the original sites which occur in groups of $k$. This leads to new effective bonds of length $k+1$ and renormalized infection rate

$$
\tilde{\lambda} / 2=\frac{\left(\lambda_{A} / 2\right)^{k+1}}{\mu^{k}} .
$$

The renormalization group step is finished after renaming $\lambda_{A} \rightarrow \lambda_{B}, \tilde{\lambda} \rightarrow \lambda_{A}$ and $\tilde{\mu} \rightarrow \mu$. Equations (7) and (8) are similar to the corresponding relations for the transverse fields and interactions in the transverse-field Ising model on generalized Fibonacci chains [32]. The main difference is the extra factor $\alpha_{k}$ in (7).

If we now iterate the renormalization group step, we obtain the following recursion relations

$$
\begin{aligned}
\lambda_{A, j+1} / 2 & =\frac{\left(\lambda_{A, j} / 2\right)^{k+1}}{\mu_{j}^{k}}, \quad \lambda_{B, j+1}=\lambda_{A, j}, \\
\mu_{j+1} & =\alpha_{k} \frac{\mu_{j}^{k+1}}{\left(\lambda_{B, j} / 2\right)^{k}}, \\
m_{j+1} & =(k+1) m_{j},
\end{aligned}
$$

where $j$ is the index of the renormalization group step. For the further analysis, it is convenient to introduce variables $R_{j}$ and $S_{j}$ that characterize the ratios of the transition rates,

$$
R_{j}=\ln \left(2 \mu_{j} / \lambda_{B, j}\right), \quad S_{j}=\ln \left(\lambda_{A, j} /\left(2 \mu_{j}\right)\right) .
$$

In terms of these variables, the recursion relations (9) and (10) turn into an inhomogeneous linear recurrence

$$
\begin{aligned}
R_{j+1} & =k R_{j}-S_{j}+A_{k}, \\
S_{j+1} & =-k R_{j}+(k+1) S_{j}-A_{k}
\end{aligned}
$$

where $A_{k}=\ln \left(\alpha_{k}\right)$. 


\section{Renormalization-group flow}

The general solution of the inhomogeneous recurrence (13)14) is the sum of a particular solution and the general solution of the corresponding homogeneous recurrence. To find a particular solution, we use the ansatz $R_{j}=$ $\bar{R}=$ const and $S_{j}=\bar{S}=$ const. Inserting this into eqs. (13) and (14) yields

$$
\bar{R}=-\frac{k-1}{k(k-2)} A_{k}, \quad \bar{S}=-\frac{1}{k(k-2)} A_{k} .
$$

The ansatz fails for the case $k=2$ which thus requires a separate calculation. It will be given in the appendix.

The general solution of the homogeneous recurrence

$$
\begin{aligned}
R_{j+1} & =k R_{j}-S_{j}, \\
S_{j+1} & =-k R_{j}+(k+1) S_{j}
\end{aligned}
$$

can be easily found by diagonalizing the coefficient matrix

$$
\mathbf{T}_{k}=\left(\begin{array}{cc}
k & -1 \\
-k & k+1
\end{array}\right)
$$

Its eigenvalues, $\zeta_{+}^{2}$ and $\zeta_{-}^{2}$, are the squares of the eigenvalues of the substitution matrix (2), and the corresponding right eigenvectors read

$$
\left(\begin{array}{c}
1 \\
-\zeta_{+}
\end{array}\right), \quad\left(\begin{array}{c}
1 \\
-\zeta_{-}
\end{array}\right)
$$

By decomposing the initial conditions $R_{0}-\bar{R}$ and $S_{0}-\bar{S}$ into the eigenvectors and multiplying with the $j$-th power of the matrix $\mathbf{T}_{k}$, we obtain the solution

$$
\begin{aligned}
R_{j} & =\frac{1}{\zeta_{+}-\zeta_{-}}\left(-\eta_{-} \zeta_{+}^{2 j}+\eta_{+} \zeta_{-}^{2 j}\right)-\frac{k-1}{k(k-2)} A_{k} \\
S_{j} & =\frac{1}{\zeta_{+}-\zeta_{-}}\left(\eta_{-} \zeta_{+}^{2 j+1}-\eta_{+} \zeta_{-}^{2 j+1}\right)-\frac{1}{k(k-2)} A_{k}
\end{aligned}
$$

The coefficients $\eta_{+}$and $\eta_{-}$are determined by the initial ratios $R_{0}$ and $S_{0}$ via

$$
\eta_{ \pm}=\zeta_{ \pm} R_{0}+S_{0}+\frac{A_{k}}{k(k-2)}\left[1+\zeta_{ \pm}(k-1)\right]
$$

Let us analyze the solution (20), (21) to find the critical point. In the limit $j \rightarrow \infty$, the behavior of $R_{j}$ and $S_{j}$ is dominated by the larger of the two eigenvalues as $R_{j} \sim-\eta_{-} \zeta_{+}^{2 j}$ and $S_{j} \sim \eta_{-} \zeta_{+}^{2 j+1}$. If $\eta_{-}$is negative, $R_{j}$ flows to $+\infty$ while $S_{j}$ flows to $-\infty$. The healing rate $\mu$ thus becomes larger than both infection rates, putting the system into the inactive phase. In contrast, if $\eta_{-}$is positive, $R_{j}$ flows to $-\infty$ while $S_{j}$ flows to $+\infty$. In this case, the system is in the active phase because the healing rate becomes smaller than both infection rates. The critical point is therefore given by the condition $\eta_{-}=0$. This can be rewritten in terms of the initial (bare) values of the transition rates as

$$
\left(\frac{2 \mu}{\lambda_{B}}\right)^{1-\zeta_{-}}=\frac{\lambda_{A}}{\lambda_{B}} \alpha_{k}^{\frac{1+\zeta_{-}(k-1)}{k(k-2)}} .
$$

\section{Critical behavior}

At criticality, $\eta_{-}=0$, the asymptotic behavior of $R_{j}$ and $S_{j}$ is determined by the smaller eigenvalue $\zeta_{-}$. Specifically,

$$
\begin{aligned}
R_{j} & =\frac{1}{\zeta_{+}-\zeta_{-}} \eta_{+} \zeta_{-}^{2 j}-\frac{k-1}{k(k-2)} A_{k}, \\
S_{j} & =-\frac{1}{\zeta_{+}-\zeta_{-}} \eta_{+} \zeta_{-}^{2 j+1}-\frac{1}{k(k-2)} A_{k} .
\end{aligned}
$$

Both quantities are negative because $\eta_{+}$and $\zeta_{-}$are negative. If $\left|\zeta_{-}\right|>1$, both $R_{j}$ and $S_{j}$ diverge towards $-\infty$ with increasing $j$, i.e, the modulation of the transition rates becomes infinitely strong. At the resulting "infinite-modulation" critical point, the condition $\lambda_{A, j} \ll \mu_{j} \ll \lambda_{B, j}$ is better and better fulfilled with increasing $j$ implying that the renormalization group becomes asymptotically exact.

To determine the critical behavior, we first analyze the flow of the inverse time scale $\Omega$ under the renormalization group. $\Omega$ can be identified with the largest transition rate in the system, $\Omega_{j}=\lambda_{B, j}$. Its recursion relation thus reads

$$
\frac{\Omega_{j}}{\Omega_{j-1}}=\frac{\lambda_{A, j-1}}{\lambda_{B, j-1}}=\exp \left(R_{j-1}+S_{j-1}\right) .
$$

Inserting the critical solutions (24) and (25), and iterating the recursion gives

$$
\Omega_{j}=\alpha_{k}^{j /(k-2)} \exp \left[\frac{\eta_{+}\left(1-\zeta_{-}\right)\left(1-\zeta_{-}^{2 j}\right)}{\left(\zeta_{+}-\zeta_{-}\right)\left(1-\zeta_{-}^{2}\right)}\right] \Omega_{0}
$$

To relate the inverse time scale $\Omega_{j}$ to the length scale $\ell_{j}$, we recall that the length of the generalized Fibonacci chain increases as $N \sim \zeta_{+}^{i}$ with inflation step $i$. As each renormalization group step corresponds to two inflation steps, this means that the length scale $\ell_{j}$ behaves as $\ell_{j} \sim$ $N \sim \zeta_{+}^{2 j}$. Inserting this relation into (27), we obtain activated dynamical scaling of the form

$$
\ln \left(\Omega_{0} / \Omega_{j}\right) \sim \ell_{j}^{\psi}
$$

The tunneling exponent is identical to the wandering exponent of the underlying Fibonacci chain, i.e., it takes the value

$$
\psi=\omega=\ln \left|\zeta_{-}\right| / \ln \zeta_{+} .
$$

We now turn to the decay of the density $\rho$ of active sites with time at criticality. Sites (clusters) that survive the renormalization group to step $j$, survive the real time evolution to time $t_{j} \sim 1 / \Omega_{j}$. The density of sites after renormalization group step $j$ is easily estimated as $\rho_{j}=$ $n_{j} m_{j}$ where $n_{j} \sim 1 / \ell_{j}$ is the density of surviving clusters and $m_{j}=(k+1)^{j}$ is their moment. Combining this with eq. (27), we obtain

$$
\rho\left(t_{j}\right) \sim\left[\ln \left(t_{j} / t_{0}\right)\right]^{-\bar{\delta}}
$$


with the critical exponent given by

$$
\bar{\delta}=\frac{1}{\psi}-\phi=\frac{1}{\psi}-\frac{\ln (k+1)}{2 \ln \left|\zeta_{-}\right|} .
$$

( $\phi$ characterizes the relation between cluster moment and inverse time scale, $m_{j} \sim\left[\ln \left(\Omega_{0} / \Omega_{j}\right)\right]^{\phi}$.)

Experiments starting from a single active seed site embedded in an otherwise inactive system can be characterized by the survival probability $P_{s}$ and the average number $N_{s}$ of sites in the active cloud. Within the renormalization group approach, a run survives to time $t$ if the seed site belongs to a cluster surviving at renormalization scale $\Omega \sim 1 / t$. As the density of (original) sites surviving after renormalization group step $j$ is given by $n_{j} m_{j}$, we find that the survival probability decays with the same critical exponent as the density, $P_{s}\left(t_{j}\right) \sim\left[\ln \left(t_{j} / t_{0}\right)\right]^{-\bar{\delta}}$. In each of the surviving runs, the number of infected sites is simply the current size of the renormalization group cluster. Thus, $N_{s}\left(t_{j}\right)=n_{j} m_{j}^{2}$. Expressing $j$ in terms of the time scale yields

$$
N_{s}\left(t_{j}\right) \sim\left[\ln \left(t_{j} / t_{0}\right)\right]^{\bar{\Theta}}
$$

with the so-called critical initial slip exponent given by

$$
\bar{\Theta}=-\frac{1}{\psi}+2 \phi=-\frac{1}{\psi}+\frac{\ln (k+1)}{\ln \left|\zeta_{-}\right|} .
$$

Note that $\bar{\Theta}, \bar{\delta}$ and $\psi$ fulfill the hyperscaling relation $\bar{\Theta}+2 \bar{\delta}-1 / \psi=0$.

Finally, we turn to the off-critical behavior. Consider a system slightly on the inactive side of the transition, $\eta_{-}<0$. According to the general solution (20), $R_{j}$ increases under renormalization. The character of the flow changes from critical to that of the inactive phase when $R_{j}$ reaches 0 . This happens at the crossover step $j^{*}$. If both $\zeta_{+}>1$ and $\left|\zeta_{-}\right|>1$, the constant term in (20) can be neglected. This yields a crossover step

$$
j^{*}=\frac{1}{2} \frac{\ln \left(\eta_{-} / \eta_{+}\right)}{\ln \left|\zeta_{-} / \zeta_{+}\right|} .
$$

The corresponding crossover length scale is given by $\ell_{j^{*}} \sim \zeta_{+}^{2 j^{*}} \sim \eta_{-}^{-\nu_{\perp}}$ with the correlation length critical exponent

$$
\nu_{\perp}=\frac{1}{1-\psi}=\frac{\ln \left(\zeta_{+}\right)}{\ln \left(\zeta_{+}\right)-\ln \left|\zeta_{-}\right|} .
$$

Interestingly, $\nu_{\perp}$ exactly saturates the Harris-Luck inequality (5).

The critical exponents $\psi, \bar{\delta}$ and $\nu$ (or, alternatively, $\psi, \bar{\Theta}$ and $\nu$ ) constitute a complete set of exponents. All other exponents can therefore be calculated from scaling relations, for example, $\beta=\bar{\delta} \nu_{\perp} \psi$.

\section{E. Log-periodic oscillations}

If the renormalized transition rates of consecutive renormalization group steps are well separated, $\mu_{j+1} \ll$ $\mu_{j}$ and $\lambda_{A, j+1} \ll \lambda_{A, j}$, the time evolution of the system proceeds in pronounced steps. For example, each downward step in density of active sites is associated with a time given by one of the renormalized decays rates, $1 / t \sim \mu_{j}$.

The generalized Fibonacci sequences are invariant under the inflation rules (11), i.e., they feature discrete scale invariance. The steps in various observables are manifestations of the log-periodic oscillations usually associated with such discrete scale invariance (see, e.g., Ref. [33] for a review).

Within the real-space renormalization group approach, the steps can be analyzed by comparing the values of an observable at two consecutive renormalization group steps. The density $\rho$ of active sites and the survival probability $P_{s}$ behave as $\rho_{j} \sim P_{s, j} \sim m_{j} / \ell_{j} \sim(k+1)^{j} \zeta_{+}^{-2 j}$. The step in $\ln \rho$ and $\ln P_{s}$ is therefore given by

$$
\Delta \ln (\rho)=\Delta \ln \left(P_{s}\right)=\ln \left[(k+1) / \zeta_{+}^{2}\right] .
$$

Because of the activated scaling, the oscillations are not $\log$-periodic but double-log periodic in time, i.e.,

$$
\Delta \ln \left[\ln \left(t / t_{0}\right)\right]=2 \ln \left|\zeta_{-}\right| .
$$

The size $N_{s}$ of the active cluster growing out of a single seed has analogous steps of magnitude

$$
\Delta \ln \left(N_{s}\right)=\ln \left[(k+1)^{2} / \zeta_{+}^{2}\right] .
$$

\section{F. Explicit predictions for $k=1,2$ and 3}

We now apply the general renormalization group theory developed above to the specific cases $k=1,2$ and 3 .

$k=1$ : Fibonacci chain. The eigenvalues of the substitution matrix $\mathbf{M}_{1}$ are given by $\zeta_{ \pm}=(1 \pm \sqrt{5}) / 2$. Their numerical values are $\zeta_{+}=1.618$ and $\zeta_{-}=-0.6180$. As $\left|\zeta_{-}\right|<1$, the (logarithmic) ratio variables $R_{j}$ and $S_{j}$ at criticality do not approach $-\infty$ under the renormalization group. Instead $R_{j}$ approaches 0 and $S_{j}$ goes to a constant. The renormalized transition rates thus eventually violate the condition $\lambda_{A} \ll \mu \ll \lambda_{B}$ (even if the bare rates fulfill it). This implies that the renormalization group method does not describe the correct asymptotic critical behavior for $k=1$.

$k=2$ : The eigenvalues of the substitution matrix $\mathbf{M}_{2}$ are $\zeta_{+}=2$ and $\zeta_{-}=-1$. As $\left|\zeta_{-}\right|=1$, the system is right at the boundary between the renormalization group method working and failing, and a more detailed analysis is required. Although the general solution (20|21) is not valid for $k=2$, we have solved this case in the appendix. At criticality, both $R_{j}$ and $S_{j}$ go towards large positive values with $j \rightarrow \infty$. This means that the renormalization group method eventually fails for $k=2$ even if the bare inhomogeneities are strong.

$k=3$ : The substitution matrix $\mathbf{M}_{3}$ has eigenvalues $\zeta_{ \pm}=(1 \pm \sqrt{13}) / 2$ with numerical values $\zeta_{+}=2.303$ and 
$\zeta_{-}=-1.303$. Because $\left|\zeta_{-}\right|>1$, the renormalization group is asymptotically exact in this case. Inserting $\zeta_{+}$ and $\zeta_{-}$into eqs. (29), (31), (33), and (35), we obtain the following values for the critical exponents: $\psi=\omega_{3}=$ $0.3171, \bar{\delta}=0.5330, \bar{\Theta}=2.086$, and $\nu=1.464$. The steps in the observables can be determined from eqs. (36) to (38) yielding $\Delta \ln (\rho)=\Delta \ln \left(P_{s}\right)=0.2819, \Delta \ln \left(N_{s}\right)=$ 1.104 , and $\Delta \ln \left[\ln \left(t / t_{0}\right)\right]=0.5290$.

$k \geq 4$ : Because $\left|\zeta_{-}\right|$increases with increasing $k$, the renormalization group method is valid for all $k \geq 4$. Critical exponents and step sizes can be calculated analogously to the $k=3$ case.

\section{MONTE-CARLO SIMULATIONS}

\section{A. Simulation method and overview}

To test the predictions of the Harris-Luck criterion and the renormalization group approach of Sec. III] we performed extensive Monte-Carlo simulations. Our system is characterized by three transition rates, the uniform healing rate $\mu$ and the infection rates $\lambda_{A}$ and $\lambda_{B}$ which are modulated according to the generalized Fibonacci chain. We set the healing rate to $\mu=1$ and tune the transition by changing $\lambda_{B}$. The ratio $\lambda_{A} / \lambda_{B}$ is treated as a fixed external parameter that determines the strength of the aperiodic inhomogeneity.

Our numerical implementation of the contact process is similar to Ref. 34] but adapted to the case of nonuniform infection rates. The algorithm starts at time $t=0$ from some configuration of infected and healthy sites and consists of a sequence of events. During each event an infected site is randomly chosen from a list of all $N_{a}$ infected sites, then a process is selected, either healing with probability $1 /\left[1+\max \left(\lambda_{A}, \lambda_{B}\right)\right]$, infection of the left neighbor with probability $(1 / 2) \lambda_{\text {left }} /[1+$ $\left.\max \left(\lambda_{A}, \lambda_{B}\right)\right]$ or infection of the right neighbor with probability $(1 / 2) \lambda_{\text {right }} /\left[1+\max \left(\lambda_{A}, \lambda_{B}\right)\right] . \quad\left(\lambda_{\text {left }}\right.$ and $\lambda_{\text {right }}$ denote the infection rates of the bonds left and right of the given site.) The infection succeeds if this neighbor is healthy. The time is then incremented by $1 / N_{a}$.

Employing this algorithm, we studied the cases $k=1,2$ and 3 using systems of up to 35 generations of the generalized Fibonacci chain (more than $10^{7}$ sites). We used several different values of the parameter characterizing the strength of the inhomogeneity, $\lambda_{A} / \lambda_{B}=$ $0.001,0.004,0.01,0.04,0.1,2 / 3,1$, and 25 . To cope with the slow dynamics at criticality, we simulated long times up to $1.4 \times 10^{9}$. Most of our simulations were spreading runs that start from a single infected seed site and measure the survival probability $P_{s}$ and the size $N_{s}$ of the active cloud. The data are averaged over up to 500,000 trials. For comparison, we have also performed a few density decay runs that start from a fully active lattice.

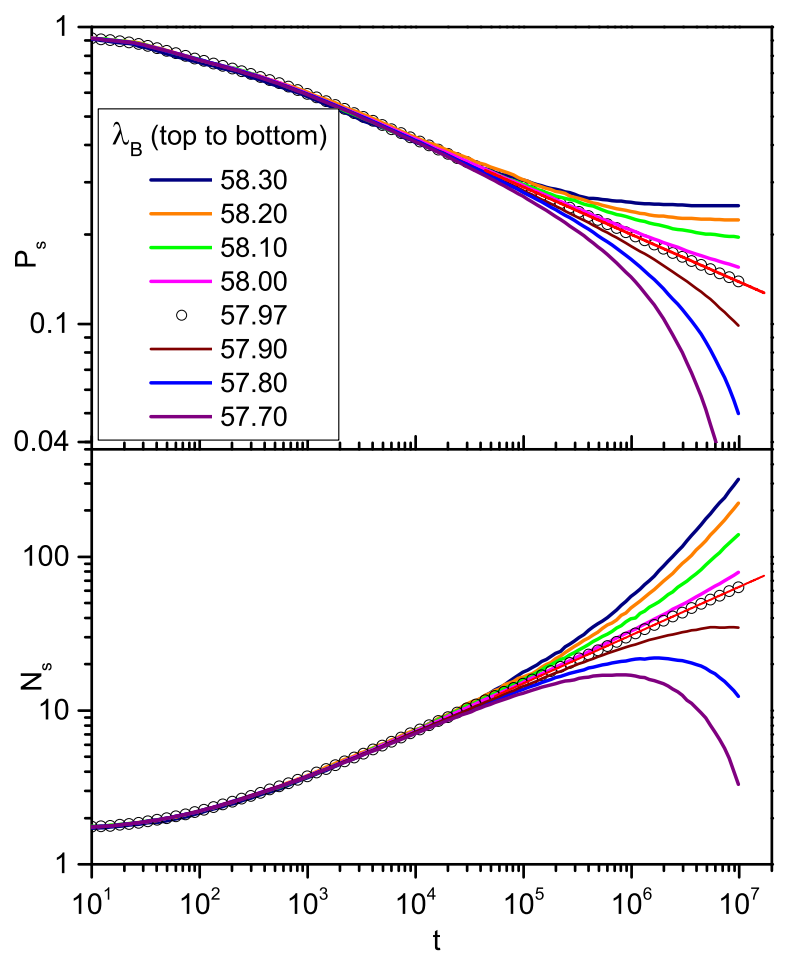

FIG. 2. (Color online) Survival probability $P_{s}$ and size $N_{s}$ of the active cloud vs. time $t$ for $k=1$ and strong inhomogeneity $\lambda_{A} / \lambda_{B}=0.01$. The data are averages of 100,000 (away from criticality) to 500,000 (at criticality) trials. The critical point is located at $\lambda_{B}=57.97$. The solid straight lines represent power-law fits giving the critical exponents $\delta=0.160$ and $\Theta=0.314$.

\section{B. Results for $k=1$}

According to the Harris-Luck criterion, weak inhomogeneities are irrelevant in the $k=1$ case because the wandering exponent $\omega_{1}=-1$ fulfills the inequality $\omega<1-1 / \nu_{\perp} \approx 0.0884$. Moreover, the renormalization group of Sec. III predicts that strong inhomogeneities decrease under renormalization. We therefore expect the contact process to display clean DP critical behavior even for strong bare inhomogeneities.

To test this prediction, we performed spreading simulations of a system having strong inhomogeneities characterized by $\lambda_{A} / \lambda_{B}=0.01$. The resulting survival probability $P_{s}$ and size $N_{s}$ of the active cloud are presented in Fig. 2. The figure shows that the critical behavior is of conventional power-law type. The critical exponents extracted from fits to $P_{s} \sim t^{-\delta}$ and $N_{s} \sim t^{\Theta}$ take the values $\delta=0.160$ and $\Theta=0.314$ in excellent agreement with the clean DP values $\delta_{\mathrm{DP}}=0.159464$ and $\Theta_{\mathrm{DP}}=0.313686$ [35]. We thus conclude that the contact process with aperiodic transition rates modulated according to the $k=1$ Fibonacci chain is indeed in the clean DP universality class. The same conclusion was reached in Ref. [36] based on simulations of the steady-state density $\rho$ for smaller 


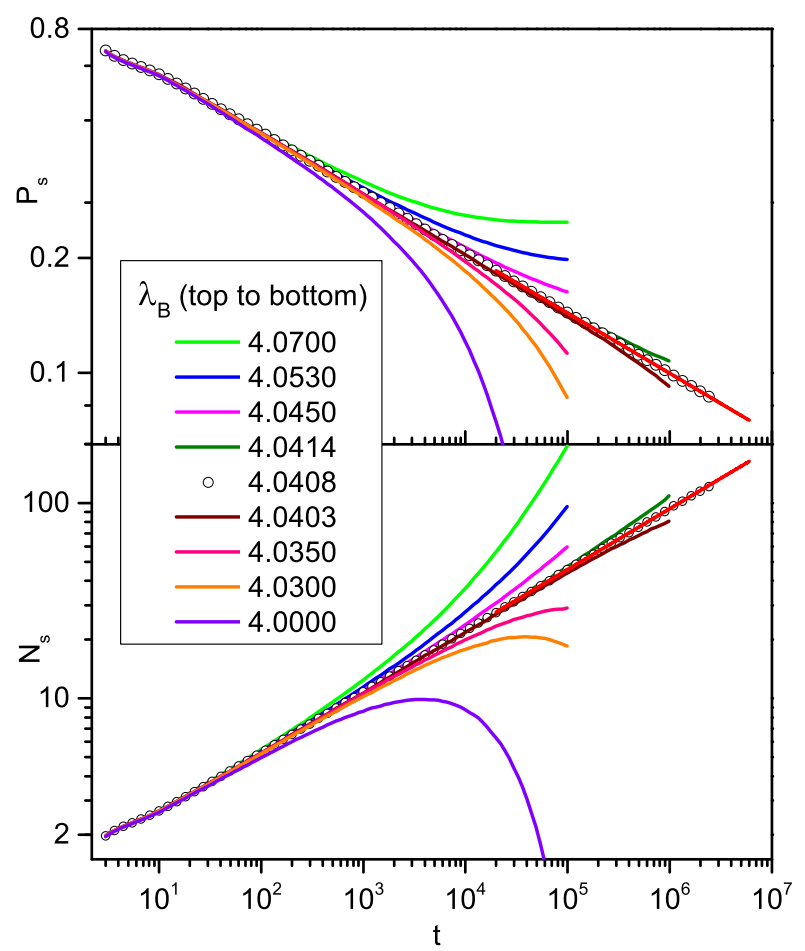

FIG. 3. (Color online) Survival probability $P_{s}$ and size $N_{s}$ of the active cloud vs. time $t$ for the case $k=2$ and weak inhomogeneity $\lambda_{A} / \lambda_{B}=2 / 3$. The data are averages of 100,000 to 150,000 trials. The critical point is located at $\lambda_{B}=4.0408$. The solid straight lines represent power-law fits giving the critical exponents $\delta=0.158$ and $\Theta=0.311$.

systems.

\section{Results for $k=2$}

The wandering exponent $\omega_{2}=0$ fulfills the HarrisLuck criterion $\omega<1-1 / \nu_{\perp} \approx 0.0884$, but just barely. This implies that the inhomogeneities are asymptotically irrelevant but their magnitude will decrease only slowly with increasing length scale. The same picture also emerges from the renormalization group solution given in the appendix: If the bare inhomogeneities are strong, the renormalization group works for a number of steps until the rates leave the region of validity $\lambda_{A} \ll \mu \ll \lambda_{B}$. For strong bare inhomogeneities, we therefore expect unconventional behavior in a transient time regime while the asymptotic behavior should be in the DP universality class. For sufficiently weak inhomogeneities, the transient regime will be missing.

To verify these predictions, we performed spreading simulations for two different inhomogeneity strengths, $\lambda_{A} / \lambda_{B}=0.01$ and $2 / 3$. Figure 3 shows the survival probability $P_{s}$ and size $N_{s}$ of the active cloud for the weak inhomogeneity case. The figure yields conventional power-law critical behavior with exponents $\delta=0.158$

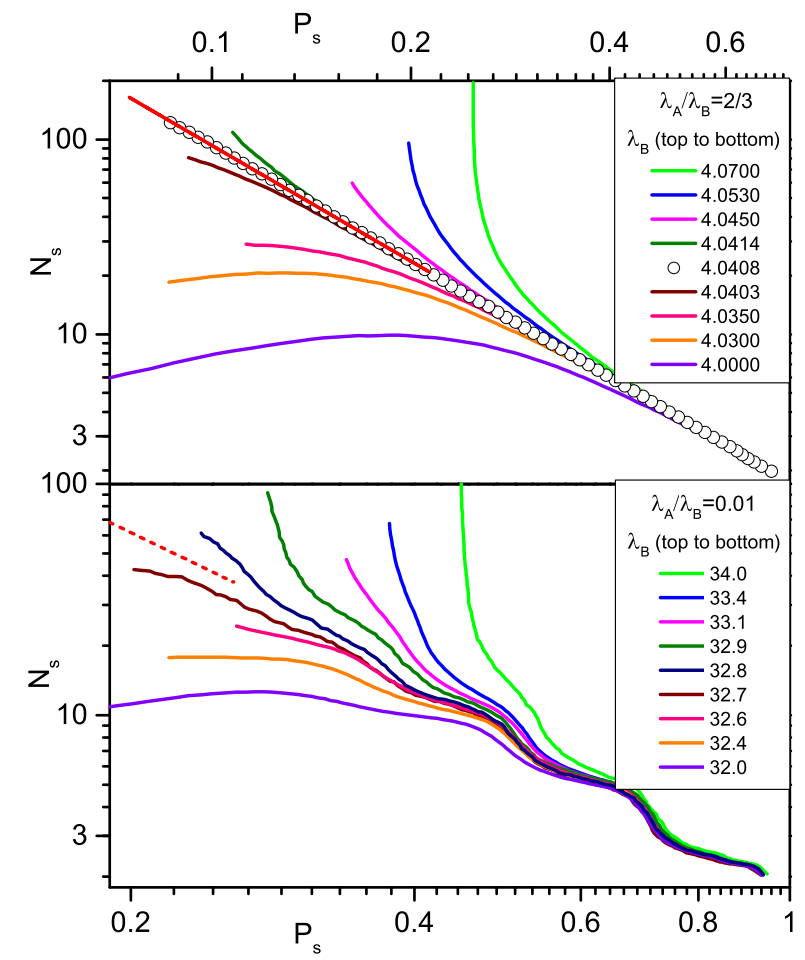

FIG. 4. (Color online) $N_{s}$ versus $P_{s}$ for the case $k=2$ and two different inhomogeneity strengths, $\lambda_{A} / \lambda_{B}=2 / 3$ (upper panel) and $\lambda_{A} / \lambda_{B}=0.01$ (lower panel). The data are averages of 100,000 to 150,000 trials. The solid line in the upper panel is a power-law fit of the critical curve $\left(\lambda_{B}=4.0408\right)$ yielding $\Theta / \delta=1.971$. The dashed line in the lower panel represents a power law with the clean exponent $-\Theta_{\mathrm{DP}} / \delta_{\mathrm{DP}}=-1.96712$.

and $\Theta=0.311$ in excellent agreement with the clean DP values $\delta_{\mathrm{DP}}=0.159464$ and $\Theta_{\mathrm{DP}}=0.313686$.

In the case of strong inhomogeneities, $\lambda_{A} / \lambda_{B}=0.01$, the behavior at early times is different as both $N_{s}$ and $P_{s}$ feature oscillations reminiscent of the steps discussed in Sec. IIIE This becomes particularly clear if one plots $N_{s}$ vs $P_{s}$ as is done in Fig. 4. The strength of the oscillations decreases with time, but only slowly. Therefore, we have not been able to reach the asymptotic regime within the available simulation times. However, the emerging critical behavior for $\lambda_{A} / \lambda_{B}=0.01$ is compatible with the clean DP universality class, as indicated by the dashed line in the lower panel of Fig. 4.

To summarize, we conclude that the asymptotic critical behavior of the $k=2$ chain is in clean DP universality class for weak inhomogeneities. The same is likely true for strong inhomogeneities. However, the asymptotic behavior is approached very slowly, giving rise to an extended transient regime of unconventional behavior that is controlled by the real-space renormalization group. 


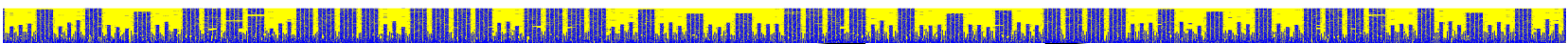

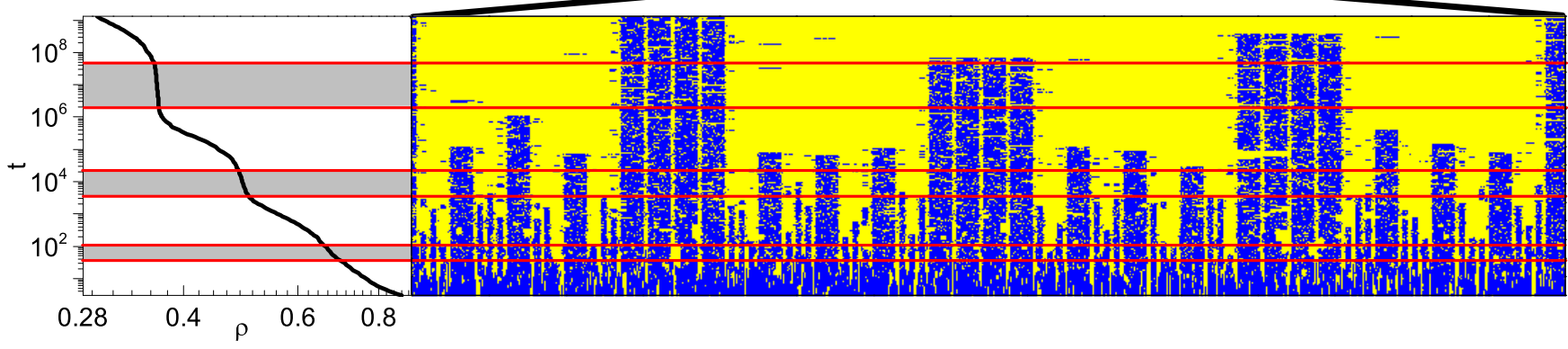

FIG. 5. (Color online) Example of a density decay run, starting from a fully active lattice of 15 generations of the $k=3$ chain (173383 sites). The inhomogeneity strength is $\lambda_{A} / \lambda_{B}=0.04$. In the main panel, dark blue dots denote active sites while light yellow marks inactive sites. The left panel shows the corresponding density $\rho$ of active sites. The horizontal lines are located at times that correspond to the inverse transition rates at different renormalization group steps, $t=\lambda_{B}^{-1}, \mu^{-1}$.

\section{Results for $k=3$}

We now turn to the case of $k=3$ for which the aperiodic inhomogeneities are relevant according to the HarrisLuck criterion. Moreover, the renormalization group theory predicts activated dynamical scaling and log-periodic or double-log periodic oscillations in various observables.

Figure 5 shows an example of a density decay run starting from a fully active lattice for an inhomogeneity strength of $\lambda_{A} / \lambda_{B}=0.04$. The figure clearly illustrates the structure of the time evolution as the system forms a hierarchy of clusters of active sites that are modulated according to the underlying $k=3$ generalized Fibonacci sequence. The corresponding time evolution of the density $\rho$ of active sites progresses in steps; in contrast to the $k=2$ case the steps become sharper and more pronounced with increasing time $t$.

To analyze the case $k=3$ quantitatively, we performed extensive spreading runs. Figure 6 shows the time evolution of $N_{s}$ and $P_{s}$ for an inhomogeneity strength $\lambda_{A} / \lambda_{B}=0.04$. Both observables show well-defined steps and plateaus as predicted in Sec. IIIE. They can also be seen in the upper panel of Fig. 7 which shows $N_{s}$ vs. $P_{s}$. In contrast to the $k=2$ case, the steps become more pronounced with increasing time. Moreover, they can be directly associated with the discrete values of the healing and infection rates appearing in the renormalization group.

From the upper panel of Fig. 7, the critical infection rate can be easily found. The critical data feature welldefined steps and plateaus while the subcritical and supercritical data curve away from the critical line as predicted. We performed analogous simulations for inhomogeneity strengths $\lambda_{A} / \lambda_{B}=0.001,0.004,0.01,0.1$ and 1 . The resulting phase diagram is shown in Fig. 8. The Monte-Carlo data are in excellent agreement with the renormalization group prediction (23) for all $\lambda_{A} / \lambda_{B} \leq 0.1$ (even though the analytical result does not contain any adjustable parameters). Surprisingly, the an-

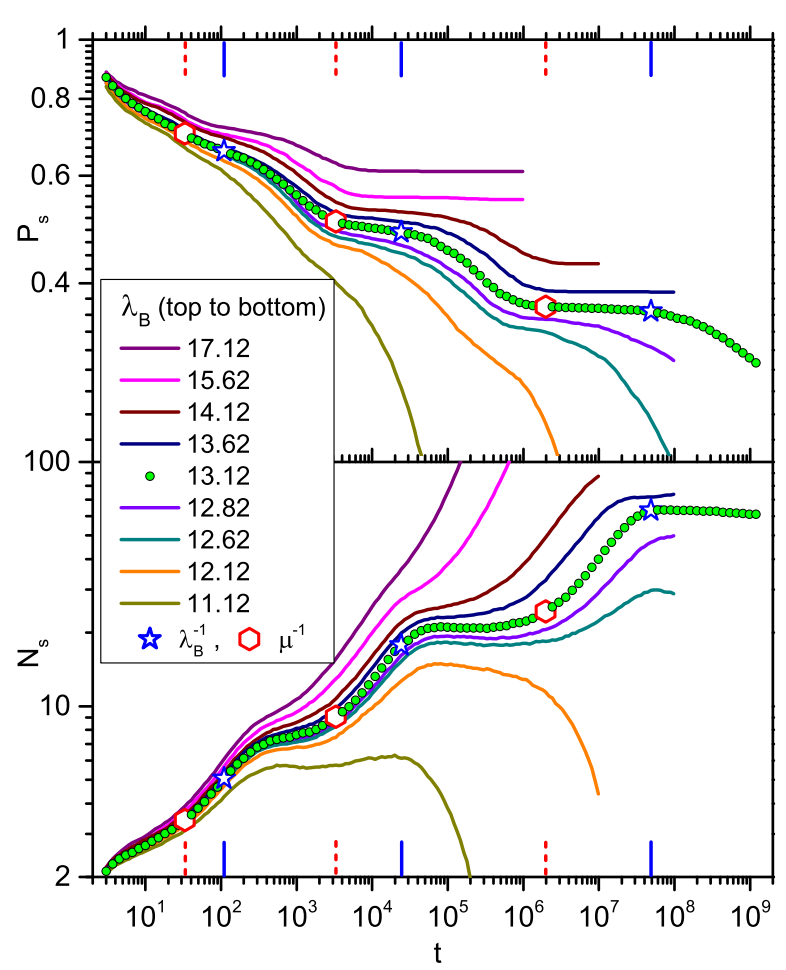

FIG. 6. (Color online) Survival probability $P_{s}$ and size $N_{s}$ of the active cloud vs. time $t$ for the case $k=3$ and $\lambda_{A} / \lambda_{B}=$ 0.04 (5000 trials). The steps and plateaus in the critical curve, $\lambda_{B}=13.12$, become more pronounced with increasing time. They can be associated with the discrete values of $\lambda$ and $\mu$ appearing in the renormalization group (marked by large stars and hexagons).

alytical result is still a good approximation in the uniform case $\lambda_{A} / \lambda_{B}=1$ where the renormalization group cannot be expected to work.

The effect of the inhomogeneity strength on the critical behavior is demonstrated in the lower panel of Fig. 7 which shows $N_{s}$ vs. $P_{s}$ for several values of $\lambda_{A} / \lambda_{B}$. If 


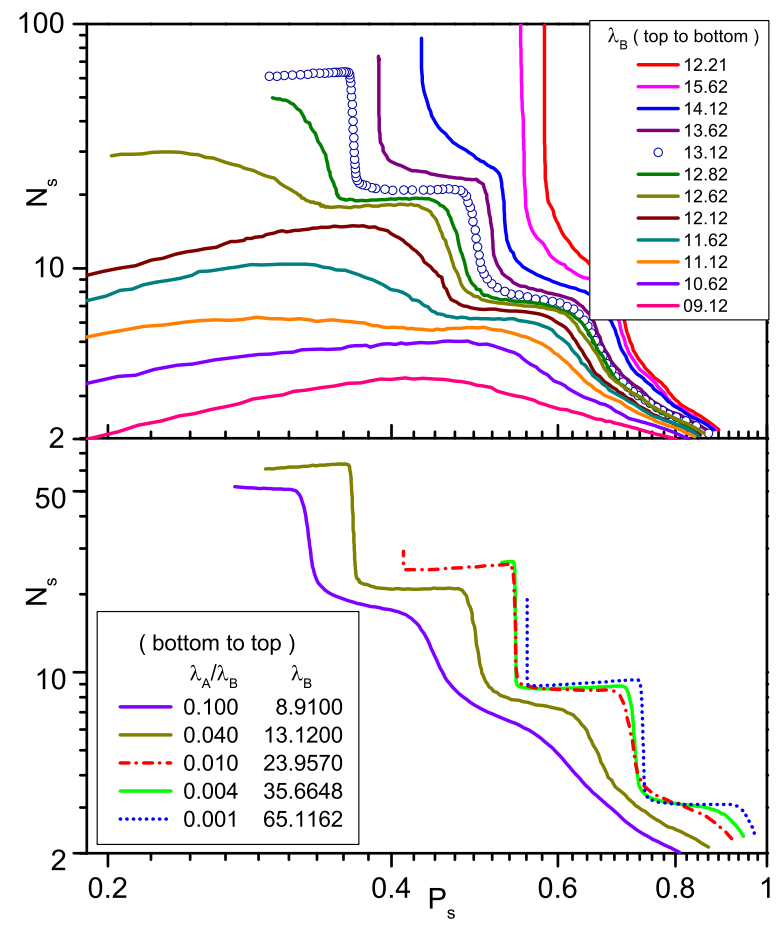

FIG. 7. (Color online) Upper panel: $N_{s}$ versus $P_{s}$ for the case $k=3$ and $\lambda_{A} / \lambda_{B}=0.04$ (5000 trials). The maximum time is $t_{\max }=1.4 \times 10^{9}$ at criticality. The critical curve, $\lambda_{B}=13.12$, shows pronounced steps as predicted in Sec. IIIE Lower panel: Critical curves for several inhomogeneity strengths $\lambda_{A} / \lambda_{B}$ (5000 to 100000 trials).

the (bare) inhomogeneities are very strong (small value of $\lambda_{A} / \lambda_{B}$ ), the steps in the critical $N_{s}$ vs $P_{s}$ curve are sharp and pronounced from the outset because the renormalization group is always in its asymptotic regime $\lambda_{A} \ll \mu \ll \lambda_{B}$. For weaker inhomogeneities, the oscillations of the $N_{s}$ vs $P_{s}$ curves are initially not very pronounced. With increasing time the steps become sharper because the renormalization group flows towards the asymptotic regime.

To compare the Monte-Carlo data and the renormalization group predictions quantitatively, we now investigate the critical $N_{s}$ vs. $P_{s}$ curve for $\lambda_{A} / \lambda_{B}=0.04$ in detail. The exponent $\bar{\Theta} / \bar{\delta}$ can be found by fitting the envelope of the $N_{s}$ vs. $P_{s}$ curve. This means fitting equivalent discrete points, each representing one renormalization group step. This analysis, shown in Fig. 9 yields $\bar{\Theta} / \bar{\delta}=3.79$. This value is in good agreement with the prediction of 3.91, in particular in view of the fact that we only have 3 steps to perform the fit. Figure 9 also allows us to determine the steps $\Delta \ln \left(P_{s}\right)$ and $\Delta \ln \left(N_{s}\right)$ between consecutive renormalization group steps. Using the data of the third step which is the last complete step in our data, we find $\Delta \ln \left(P_{s}\right)=0.284$ and $\Delta \ln \left(N_{s}\right)=1.092$, again in good agreement with the renormalization group predictions of Sec. IIIE 0.2819 and 1.104 , respectively

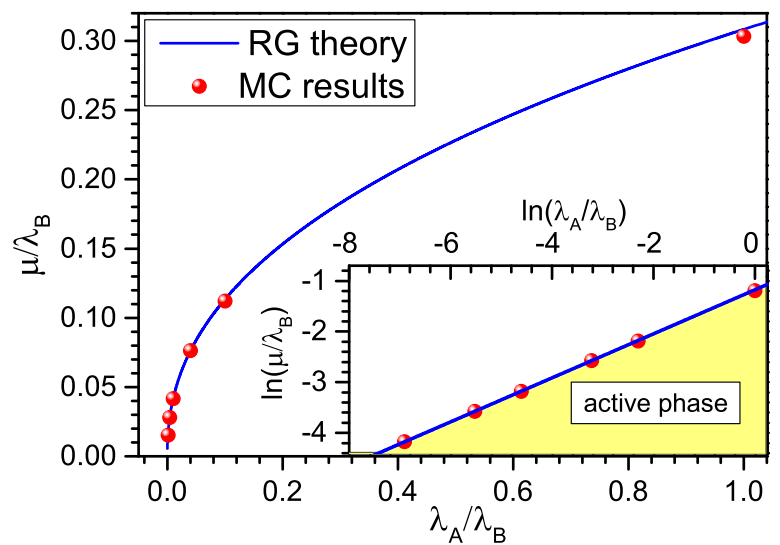

FIG. 8. (Color online) Phase diagram of the contact process for $k=3$. The dots are the Monte-Carlo results for $\lambda_{A} / \lambda_{B}=$ $0.001,0.004,0.01,0.04,0.1$, and 1 . The solid line represents the renormalization group result (23).

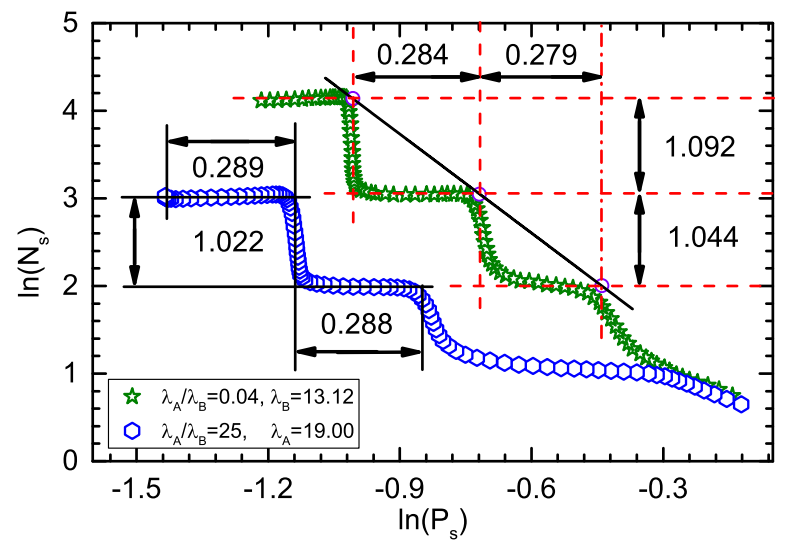

FIG. 9. (Color online) Quantitative analysis of the critical $N_{s}$ versus $P_{s}$ curve for $k=3$ and $\lambda_{A} / \lambda_{B}=0.04$ (maximum time $t_{\max }=1.4 \times 10^{9}$ ) and $\lambda_{A} / \lambda_{B}=25$ (maximum time $\left.t_{\max }=2 \times 10^{8}\right)$. The solid line is a fit of the envelop of the curve to the power law $N_{s} \sim P_{s}^{-\bar{\Theta} / \bar{\delta}}$ yielding $\bar{\Theta} / \bar{\delta}=3.79$.

The renormalization group results of Sec. III were derived under the assumption $\lambda_{A} \ll \mu \ll \lambda_{B}$. It is important to investigate whether the resulting renormalization group fixed point attracts the flow from a larger part of parameter space. In other words, is the asymptotic critical behavior controlled by this fixed point even if the bare system violates the condition $\lambda_{A} \ll \mu \ll \lambda_{B}$. In the lower panel of Fig. 7, we have seen that the fixed point attracts the flow from regions where $\lambda_{A} / \lambda_{B}$ is only moderately small. We now look at an extreme case in which the bare system strongly violates the condition. The second curve in Fig. 9 shows the critical $N_{s}$ vs $P_{s}$ data for $\lambda_{A} / \lambda_{B}=25$. As expected, the two curves initially behave differently. However, the steps $\Delta \ln \left(P_{s}\right)$ and $\Delta \ln \left(N_{s}\right)$ forming at later times appear to be iden- 
tical within the numerical errors. Moreover, we also performed density decay runs for $\lambda_{A} / \lambda_{B}=25$. A figure (not shown) analogous to Fig. 5 clearly demonstrates that the same hierarchy of clusters forms at late times for $\lambda_{A} / \lambda_{B}=0.04$ and $\lambda_{A} / \lambda_{B}=25$. This strongly suggests that the renormalization group fixed point discovered in Sec. III also describes the critical behavior of the system with $\lambda_{A} / \lambda_{B}=25$.

\section{CONCLUSIONS}

In summary, we have studied the one-dimensional contact process with aperiodically modulated transition rates by means of a real-space renormalization group and by Monte-Carlo simulations. We have focused on modulations according to three cases $k=1,2,3$ of the generalized Fibonacci sequence defined by the inflation rules $\mathrm{A} \rightarrow \mathrm{AB}^{k}$ and $\mathrm{B} \rightarrow \mathrm{A}$. For $k=1$ (the Fibonacci chain proper), the inhomogeneities are strongly irrelevant according to the Harris-Luck criterion at the clean DP critical point. Correspondingly, our numerical simulations yield critical behavior in the clean DP universality class even if the initial inhomogeneities are strong. This agrees with earlier results on the steady state density [36].

In the $k=2$ case, the inhomogeneities are still irrelevant at the clean DP critical point, but just barely. This implies that their scale dimension is close to zero. The inhomogeneity strength therefore decreases only slowly with increasing length and time scales. Our Monte-Carlo simulations confirm this picture. If the (bare) inhomogeneities are weak, we again find critical behavior in the clean DP universality class. For strong inhomogeneities, the system shows unconventional behavior in an extended transient time regime that is controlled by the real-space renormalization group. The long-time evolution appears to approach the clean DP critical behavior. However, we could not reach the true asymptotic regime within our simulation time for strong inhomogeneities.

For $k \geq 3$, the aperiodic modulation of the transition rates is relevant at the clean DP critical point because the Harris-Luck criterion is violated. We have developed a renormalization group theory of the transition and identified a fixed point that describes unconventional criticality. At this infinite-modulation fixed point, the inhomogeneity strength diverges, and the method becomes asymptotically exact. The resulting critical behavior is characterized by activated dynamical scaling. Moreover, the time dependence of observables such as the density of active sites, the survival probability, and the size of the active cloud show striking plateaus and steps. They are a consequence of the discrete scale invariance of the generalized Fibonacci sequence and related to the log-periodic oscillations found in many aperiodic systems (see, e.g., [33]). Due to the activated dynamical scaling, the oscillations are actually double-log periodic in time. Analogous double-log oscillations should occur in other systems featuring activated scaling, for example in quantum spin chains [37].

We have numerically confirmed these renormalization group predictions for the case $k=3$. The Monte-Carlo simulations also provide evidence for the critical behavior to be universal, i.e., it is valid for both weak and strong aperiodic modulations.

It is interesting to compare the phase transitions in the aperiodic contact process (for $k \geq 3$ ) and the disordered contact process. In both cases, the fluctuations of the transition rates at criticality diverge with increasing length scale. In the disordered contact process, this leads to an infinite-randomness critical point [23, 38]; and for aperiodic rates, the critical point is of infinite-modulation type. Both these critical points feature unconventional activated dynamical scaling rather than the usual powerlaw scaling. In the disordered case, the critical point is accompanied by Griffiths singularities 24, 39, 40] which are missing in the aperiodic case because the generalized Fibonacci chains do not have rare regions. Conversely, the log-periodic oscillations of observables in the aperiodic chain do not exist in the disordered chain because the latter system has continuous rather than discrete scale invariance (in the statistical sense).

Our renormalization group method is similar to the approach used in Ref. 32 to study the aperiodic transversefield Ising chain. In fact, the critical behavior of the contact process and the transverse-field Ising chain are identical in the cases in which the renormalization group correctly describes the critical point (i.e., $k \geq 3$ ). This mirrors the behavior of the corresponding random systems: The random transverse-field Ising chain [41] and the random contact process [23] feature the same critical exponents.

The main difference between the Ising chain and the contact process occurs for $k=2$. For the Ising chain, $k=2$ aperiodic modulations are exactly marginal according to the Harris-Luck criterion. This is reflected in the fact that the modulation strength stays constant under the renormalization group, leading to nonuniversal critical behavior [32]. In contrast, $k=2$ aperiodic modulations of the contact process are weakly irrelevant. Correspondingly, the renormalization group works at best in a transient time regime while the asymptotic critical behavior appears to be in the clean DP universality class.

Recently, aperiodic sequences were used to construct complex networks with long-range connections; and the contact process on such networks was studied [42]. The nonequilibrium phase transition features power-law critical behavior with exponents that depend on the underlying network. Time-dependent quantities exhibit logperiodic oscillations due to the discrete scale invariance of the networks.

Let us finally comment on generalizations to higher dimensions. One could, for example, construct higherdimensional aperiodic modulations of the transition rates by repeating identical one-dimensional sequences in the second (and third) direction. This would increase the relevance of the modulations in the Harris-Luck criterion 
because the clean correlation length exponent decreases with increasing dimension while the fluctuations of the distance to criticality are unchanged. In the random case, such correlated inhomogeneities lead to a smearing of the DP critical point [43] because rare regions undergo the transition independently. As the aperiodic systems do not have any rare regions, their behavior is likely different Alternatively, one could also look at more general tilings in two and three dimensions. Of particular interest are structures with unbounded fluctuations such as the tiling proposed in Ref. [44]. Studying the contact process on such lattices remains a task for future.

\section{ACKNOWLEDGEMENTS}

This work has been supported in part by the NSF under grants no. DMR-0906566, DMR-1205803, and PHYS-1066293.

\section{APPENDIX: THE CASE $k=2$}

The general solution of the renormalization group developed in Sec. IIIC does not apply to the case $k=2$ because the particular solution of the inhomogeneous recurrence (13) and (14) is not of the form $R_{j}=\bar{R}=$ const and $S_{j}=\bar{S}=$ const. The reason is that the smaller eigenvalue of the coefficient matrix $\mathbf{T}_{2}$ takes the value $\zeta_{-}^{2}=1$.
In this appendix, we therefore directly solve the problem for $k=2$. After introducing the variables $X_{j}=$ $2 R_{j}+S_{j}$ and $Y_{j}=R_{j}-S_{j}$ into (13) and (14), the recurrence relations read

$$
\begin{aligned}
X_{j+1} & =X_{j}+A, \\
Y_{j+1} & =4 Y_{j}+2 A .
\end{aligned}
$$

As the two equations are now decoupled, they can be easily solved,

$$
\begin{aligned}
X_{j} & =X_{0}+j A \\
Y_{j} & =-\frac{2}{3} A+4^{j}\left(Y_{0}+\frac{2}{3} A\right)
\end{aligned}
$$

where $X_{0}=2 R_{0}+S_{0}$ and $Y_{0}=R_{0}-S_{0}$. Transforming back to the variables $R_{j}$ and $S_{j}$, we finally obtain

$$
\begin{aligned}
R_{j} & =\frac{1}{3}\left[X_{0}+j A-\frac{2}{3} A+4^{j}\left(Y_{0}+\frac{2}{3} A\right)\right], \\
S_{j} & =\frac{1}{3}\left[X_{0}+j A+\frac{4}{3} A-2 \times 4^{j}\left(Y_{0}+\frac{2}{3} A\right)\right] .
\end{aligned}
$$

For $Y_{0}+2 A / 3>0$, the system is in the inactive phase because $R_{j} \rightarrow \infty$ and $S_{j} \rightarrow-\infty$ under the renormalization group. In contrast, the system is in the active phase for $Y_{0}+2 A / 3<0$. At criticality, $Y_{0}+2 A / 3=0$, both $R_{j}$ and $S_{j}$ increase linearly with $j$. The implies that the renormalization group method asymptotically fails because the transition rates eventually violate the condition $\lambda_{A} \ll \mu \ll \lambda_{B}$.
[1] V. P. Zhdanov and B. Kasemo, Surface Science Reports 20, 113 (1994).

[2] B. Schmittmann and R. K. P. Zia, in Phase Transitions and Critical Phenomena, Vol. 17, edited by C. Domb and J. L. Lebowitz (Academic, New York, 1995) p. 1.

[3] J. Marro and R. Dickman, Nonequilibrium Phase Transitions in Lattice Models (Cambridge University Press, Cambridge, 1999).

[4] H. Hinrichsen, Adv. Phys. 49, 815 (2000).

[5] G. Odor, Rev. Mod. Phys. 76, 663 (2004)

[6] S. Lübeck, Int. J. Mod. Phys. B 18, 3977 (2004).

[7] U. C. Täuber, M. Howard, and B. P. Vollmayr-Lee, J. Phys. A 38, R79 (2005).

[8] M. Henkel, H. Hinrichsen, and S. Lübeck, Nonequilibrium phase transitions. Vol 1: Absorbing phase transitions (Springer, Dordrecht, 2008).

[9] P. Grassberger and A. de la Torre, Ann. Phys. (NY) 122, 373 (1979)

[10] H. K. Janssen, Z. Phys. B 42, 151 (1981).

[11] P. Grassberger, Z. Phys. B 47, 365 (1982).

[12] T. E. Harris, Ann. Prob. 2, 969 (1974)

[13] K. A. Takeuchi, M. Kuroda, H. Chate, and M. Sano, Phys. Rev. Lett. 99, 234503 (2007).

[14] L. Corte, P. M. Chaikin, J. P. Gollub, and D. J. Pine, Nature Physics 4, 420 (2008).

[15] A. Franceschini, E. Filippidi, E. Guazzelli, and D. J.
Pine, Phys. Rev. Lett. 107, 250603 (2011)

[16] S. Okuma, Y. Tsugawa, and A. Motohashi, Phys. Rev. B 83, 012503 (2011)

[17] H. K. Janssen, Phys. Rev. E 55, 6253 (1997).

[18] A. B. Harris, J. Phys. C 7, 1671 (1974)

[19] M. Bramson, R. Durrett, and R. H. Schonmann, Ann. Prob. 19, 960 (1991).

[20] A. G. Moreira and R. Dickman, Phys. Rev. E 54, R3090 (1996); R. Dickman and A. G. Moreira, ibid. 57, 1263 (1998).

[21] I. Webman, D. B. Avraham, A. Cohen, and S. Havlin, Phil. Mag. B 77, 1401 (1998).

[22] R. Cafiero, A. Gabrielli, and M. A. Munoz, Phys. Rev. E 57, 5060 (1998).

[23] J. Hooyberghs, F. Iglói, and C. Vanderzande, Phys. Rev. Lett. 90, 100601 (2003); Phys. Rev. E 69, 066140 (2004).

[24] A. J. Noest, Phys. Rev. Lett. 57, 90 (1986); Phys. Rev. B 38, 2715 (1988).

[25] T. Vojta and M. Dickison, Phys. Rev. E 72, 036126 (2005).

[26] M. M. de Oliveira and S. C. Ferreira, J. Stat. Mech. 2008, P11001 (2008).

[27] T. Vojta, A. Farquhar, and J. Mast, Phys. Rev. E 79, 011111 (2009); T. Vojta, Phys. Rev. E 86, 051137 (2012). 
[28] T. Vojta and M. Y. Lee, Phys. Rev. Lett. 96, 035701 (2006); M. Y. Lee and T. Vojta, Phys. Rev. E 79, 041112 (2009).

[29] J. M. Luck, EPL (Europhysics Letters) 24, 359 (1993).

[30] R. Moody, ed., The Mathematics of Long-range Aperiodic Order (Kluwer, Dordrecht, 1997).

[31] This is not a real restriction as the renormalization group steps of Sec. III alternate between modulated infection and healing rates.

[32] F. J. O. Filho, M. S. Faria, and A. P. Vieira, J. Stat. Mech. 2012, P03007 (2012).

[33] D. Sornette, Physics Reports 297, 239 (1998)

[34] R. Dickman, Phys. Rev. E 60, R2441 (1999).
[35] I. Jensen, J. Phys. A 32, 5233 (1999).

[36] M. S. Faria, D. J. Ribeiro, and S. R. Salinas, J. Stat. Mech. 2008, P01022 (2008)

[37] A. P. Vieira, Phys. Rev. Lett. 94, 077201 (2005)

[38] F. Igloi and C. Monthus, Phys. Rep. 412, 277 (2005).

[39] R. B. Griffiths, Phys. Rev. Lett. 23, 17 (1969).

[40] T. Vojta, J. Phys. A 39, R143 (2006)

[41] D. S. Fisher, Phys. Rev. B 51, 6411 (1995)

[42] R. Juhász and G. Ódor, Phys. Rev. E 80, 041123 (2009)

[43] T. Vojta, Phys. Rev. E 70, 026108 (2004); M. Dickison and T. Vojta, J. Phys. A 38, 1199 (2005).

[44] C. Godrèche and F. Lançon, J. Phys. I France 2, 207 (1992). 